\title{
FASCIOLASIS EN NIÑOS DE TRES DISTRITOS DEL DEPARTAMENTO DE JUNÍN, PERÚ
}

\author{
Fasciolasis in Children of Three Districts of Junin, Peru
}

\author{
Carina Mantari T. ${ }^{1}$, Amanda Chávez V. ${ }^{1,4}$, Francisco Suárez A. ${ }^{2}$, Carlos Arana D. ${ }^{3}$, \\ Rosa Pinedo V. ${ }^{1}$, Rosendo Ccenta E. ${ }^{1}$
}

\section{RESUMEN}

El objetivo del estudio fue determinar la prevalencia de fasciolasis ocasionada por Fasciola hepatica en niños de tres distritos del departamento de Junín, Perú, mediante exámenes coprológicos y analizar su asociación con las variables edad, género y lugar de procedencia. Se colectaron 312 muestras fecales a niños de 4 a 12 años de edad de los distritos de Masma ( $n=128)$ y Llocllapampa $(n=70)$, provincia de Jauja, y del distrito de Santa Rosa de Ocopa $(n=114)$, provincia de Concepción, entre agosto y setiembre de 2009. Las muestras fueron analizadas mediante la técnica coprológica de sedimentación rápida. Se encontró una prevalencia de $11.7 \%$ en Masma y de $0.9 \%$ en Santa Rosa de Ocopa $(\mathrm{p}<0.05)$, mientras que las muestras del distrito de Llocllapampa resultaron negativas. No se encontró asociación significativa con la infección por efecto del género o la edad de los niños. Los hallazgos confirman la persistencia de la infección en algunas zonas del departamento de Junín.

Palabras clave: distoma hepático, fasciolasis, Jauja, sedimentación, zoonosis

\section{Abstract}

The objective of this study was to determine the prevalence of fasciolasis caused by Fasciola hepatica in children of three districts of Junin, Peru and its association with age, gender and location. It was collected 312 faecal samples from children aged 4 to 12 years in the Masma $(n=128)$ and Llocllapampa $(n=70)$ districts, province of Jauja, and Santa Rosa de Ocopa district $(\mathrm{n}=114$ ), province of Concepcion, in August and September, 2009. Samples were analyzed by the fast sedimentation coprologic technique. The results showed a prevalence of $11.7 \%$ in Masma and $0.9 \%$ in Santa Rosa de Ocopa $(\mathrm{p}<0.05)$, whereas all samples from Llocllapampa district were negative. There was not statistical association between gender and age with presence of infection. These findings confirm the persistence of the infection in some areas of Junin.

Key words: liver fluke, fasciolasis, Jauja, sedimentation, zoonosis

${ }^{1}$ Laboratorio de Parasitología y Microbiología Veterinaria, ${ }^{2}$ Laboratorio de Medicina Veterinaria Preventiva, Facultad de Medicina Veterinaria, Universidad Nacional Mayor de San Marcos, Lima

${ }^{3}$ Estación Experimental del Centro de Investigación IVITA - El Mantaro, Huancayo

${ }^{4}$ E-mail: achavezvg@gmail.com 


\section{INTRODUCCIÓN}

La fasciolasis, parasitosis causada por los tremátodos Fasciola hepatica y $F$. gigantica, presenta la más amplia distribución latitudinal, longitudinal y altitudinal conocida (Mas-Coma, 2004), notificándose desde 1950 en 51 países, con estimaciones que van desde 2.4 hasta 17 millones de personas infectadas (Esteban et al., 1999; Mas-Coma et al., 1999) y de 91 millones de personas en riesgo de infección en el mundo (Keiser y Utzinger, 2005). El mayor número de individuos infectados ha sido reportado en Bolivia, Cuba, Egipto, Perú y la República Islámica de Irán (OMS, 1995). Asimismo, se encuentra como enfermedad emergente en el sureste asiático, de acuerdo con el alto número de casos diagnosticados en Vietnam (De et al., 2006).

En el Perú, la fasciolasis, ocasionada por $F$. hepatica, constituye la segunda enfermedad parasitaria de importancia en la ganadería (Leguía, 1991). Las pérdidas económicas se estiman en cerca de 50 millones de dólares americanos por año, debido a la disminución de la ganancia de peso, reducida fertilidad y productividad y a los costos asociados con el tratamiento; sin embargo, es difícil estimar el impacto económico de la fasciolasis humana, cuyas consecuencias y secuelas de infección no solo tienen un impacto económico, sino, además, un impacto negativo en el desarrollo y salud de los pacientes infectados (Espinoza et al., 2010).

Así también, y debido a un incremento significativo de casos reportados en las últimas cuatro décadas, la fasciolasis humana es considerada una enfermedad infecciosa parasitaria emergente (Marcos et al., 2007), siendo los niños en edad escolar (5-16 años) el grupo más expuesto y cuyas tasas de infección son las más altas. En varios distritos del departamento de Junín, Perú, se han reportado prevalencias elevadas; $28.5 \%$ en Huertas (Terashima, 1970), 34.2\% en Santa Ana (Stork et al., 1973) y 18.3 y $12.6 \%$ en
Huertas y Julcán, respectivamente (Marcos et al., 2004); lo cual indica que las medidas de control y prevención no vienen dando los resultados esperados (Castro, 2007; Marcos et al., 2007).

Desafortunadamente, existen algunas dificultades inevitables en el diagnóstico de la fasciolasis humana en áreas endémicas de países en desarrollo. Las pruebas indirectas o pruebas inmunológicas, ya sean serológicas o mediante coproantígenos, no resultan muy apropiadas para la estimación de intensidad debido a los altos costos en reactivos y por requerir equipos de laboratorio de alto costo. Por consiguiente, el análisis coprológico, a pesar de la baja sensibilidad en la detección de huevos en muestras fecales y su limitación para el diagnóstico de pacientes en fase aguda, continúa siendo la estrategia de diagnóstico más apropiada para la detección de infección y la estimación de carga parasitaria; permitiendo así el diagnóstico y, con ello, el avance en el tratamiento de esta parasitosis (Valero et al., 2009).

El presente estudio pretende determinar los índices actuales de infección a $F$. hepatica en niños de algunas zonas ganaderas del departamento de Junín, a fin de poder establecer medidas correctivas de control y prevención.

\section{MATERIALES y MéTodos}

\section{Lugar de Estudio}

El estudio se realizó entre agosto y setiembre de 2009 en los distritos de Masma y Llocllapampa, provincia de Jauja, y en el distrito de Santa Rosa de Ocopa, provincia de Concepción, ambos en el departamento de Junín.

Los indicadores sociodemográficos muestran un 65,39 y $9 \%$ de población rural en los distritos de Masma, Llocllapampa y Santa Rosa de Ocopa (INEI, 2007). 


\section{Participación de la Población}

Se llevaron a cabo charlas informativas en los centros educativos de nivel primario de cada localidad y en presencia de los profesores y alumnado en general. Se explicaron los aspectos básicos de la enfermedad y el motivo de la investigación, y se entregó a los padres y tutores un documento con los detalles de la investigación para su firma de aceptación de participación voluntaria en el estudio. Además, se les entregó frascos plásticos con las indicaciones necesarias para la toma de muestras.

Asimismo, las autoridades de los Centros de Salud de los tres distritos fueron partícipes de los objetivos y resultados del estudio.

\section{Tamaño Muestral}

Se determinó mediante la fórmula para estimar proporción de poblaciones infinitas (Daniel, 1996), utilizando una prevalencia referencial del 28.3\% (Marcos et al., 2004). El tamaño mínimo resultante fue de 312. Por otro lado, se utilizó los datos de la población del Censo Nacional (INEI, 2007) y la fórmula de estratificación (Pérez, 2000) para hallar el número de muestras por distrito, siendo de 128,70 y 114 muestras para los distritos de Masma, Llocllapampa y Santa Rosa de Ocopa, respectivamente.

\section{Muestras de Heces y Análisis}

Las muestras fecales fueron tomadas por conveniencia dentro de la población referente. Los envases de plástico para la toma de la muestra de heces eran de $200 \mathrm{ml}$, de boca ancha y tapa rosca, y se solicitó a los padres y tutores de los niños que la muestra fuera no menos de $8 \mathrm{~g}$ de heces libres de contacto de tierra y orina. Se recolectó una muestra por sujeto.

Las muestras fueron conservadas en formol al $10 \%$, a razón de una parte de heces por tres partes de fijador, para el pro- cesamiento y análisis coprológico en el Laboratorio de Parasitología de la Estación Experimental IVITA-El Mantaro, Huancayo, y en el Laboratorio de Parasitología de la Facultad de Medicina Veterinaria de la Universidad Nacional Mayor de San Marcos, Lima.

Para la evaluación coproparasitológica se utilizó la técnica de sedimentación rápida modificada por Lumbreras (Lumbreras et al., 1962). El diagnóstico de la infección por $F$. hepatica se consideró positivo cuando se encontró huevos compatibles con las características estructurales de la especie (WHO, 1991) (Fig. 1).

\section{Análisis Estadístico}

La prevalencia de fasciolasis se expresó en forma porcentual de acuerdo a los resultados parasitológicos, con sus respectivos intervalos de confianza al $95 \%$. Se evaluaron las variables género, lugar de procedencia y edad. La posible asociación entre las variables de interés y la presencia de huevos de $F$. hepatica se analizó mediante la prueba de Chi Cuadrado. Los datos se procesaron mediante el paquete estadístico SPSS v. 10.0, estableciendo la significación estadística en en $\mathrm{p}<0.05$.

\section{Resultados}

La prevalencia global de F. hepatica en los tres distritos fue de $5.1 \pm 2.4 \%$ (Cuadro 1). En los distrito de Masma y Santa Rosa de Ocopa se encontraron prevalencias de 11.7 y $0.9 \%$, respectivamente, mientras que no se encontraron muestras positivas en el distrito de Llocllapampa. El distrito de Masma fue el distrito de mayor riesgo para la presentación de la enfermedad $(p<0.05)$. Por otro lado, no se encontró diferencia significativa entre ambos géneros ni entre grupos etarios (Cuadro 1).

En la prueba coprológica se detectaron, además del F. hepatica, otros parásitos in 
Cuadro 1. Prevalencia de Fasciola hepatica en niños de tres distritos de las provincias de Concepción y Jauja, Junín, mediante la técnica de sedimentación rápida (agostosetiembre, 2009)

\begin{tabular}{llccc}
\hline \multirow{2}{*}{ Variable } & & \multicolumn{2}{c}{ Muestras } & Prevalencia \pm I.C. $^{1}$ \\
\cline { 3 - 5 } & & Total (n) & Positivas (n) & \\
\hline Distrito & Santa Rosa de Ocopa & 114 & 1 & $0.9 \pm 1.7$ \\
& Masma & 128 & 15 & $11.7 \pm 5.6$ \\
& Llocllapampa & 70 & 0 & 0 \\
Género & Masculino & 142 & 9 & $6.3 \pm 4.0$ \\
& Femenino & 170 & 7 & $4.1 \pm 3.0$ \\
\multirow{2}{*}{ Edad (años) } & $4-7$ & 118 & 5 & $4.2 \pm 3.6$ \\
& $8-12$ & 194 & 11 & $5.2 \pm 3.1$ \\
\hline Total & & 312 & 16 & $5.1 \pm 2.4$ \\
\hline
\end{tabular}

${ }^{1}$ Intervalo de confianza del 95\%

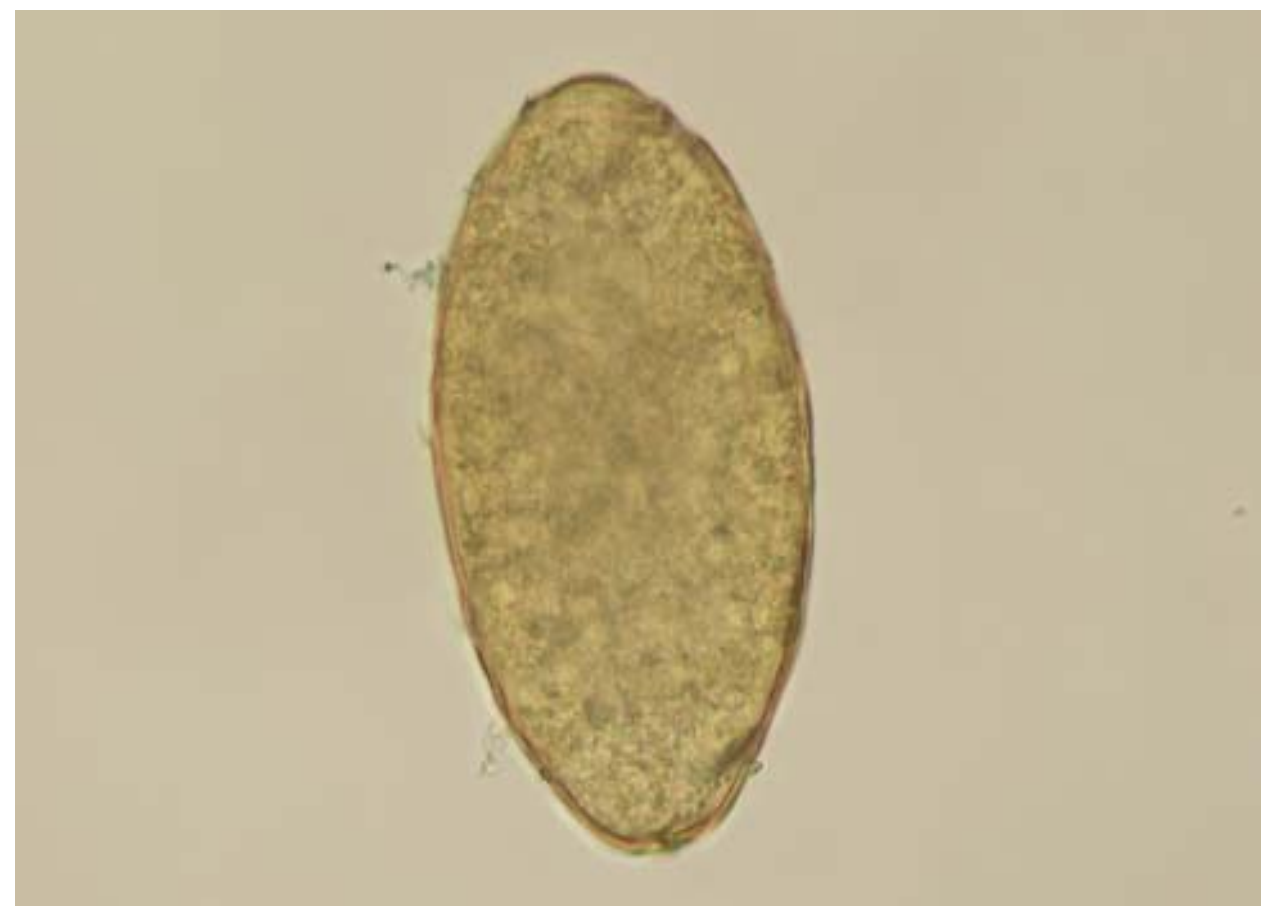

Figura 1. Huevo de Fasciola hepatica (145.8 x $75.6 \mu \mathrm{m}, 40 \mathrm{x})$ hallado en la muestra de un niño del distrito de Masma, Jauja, Junín 
testinales como Ascaris lumbricoides, Trichuris spp, Hymenolepis nana y Entamoeba coli, con una tasa de infección de $50.8,22.8$ y $14.3 \%$ para los distritos de Masma, Santa Rosa de Ocopa y Llocllapampa, respectivamente.

\section{Discusión}

La fasciolasis es un importante problema de salud pública en el Perú debido a la alta prevalencia de infección humana, especialmente en niños (Esteban et al., 2002; Marcos et al., 2005) y un problema veterinario, pues constituye una de las tres enfermedades parasitarias más importantes que afecta a la ganadería en el Perú (Leguía, 1997; Espinoza et al., 2010).

Estudios epidemiológicos realizados en el valle del Mantaro, zona del presente estudio la señalan como hiperendémica (Marcos et al., 2004); es decir, con prevalencias de fasciolasis mayores de 10\% (Mas-Coma et al., 1999). Los tres distritos evaluados presentaban condiciones favorables para el desarrollo de la distomatosis hepática, tales como temperatura $\left(10\right.$ a $\left.22{ }^{\circ} \mathrm{C}\right)$, humedad adecuada y presencia de fuentes de agua; sin embargo, los resultados obtenidos mediante la prueba de sedimentación rápida fueron muy disímiles entre distritos, imposibilitando realizar una clasificación global al departamento de Junín.

La presencia de charcos, riachuelos y pantanos cercanos a las viviendas y colindantes con campos de cultivo y áreas de pastoreo, así como la presencia de plantas acuáticas (berros) en el distrito de Masma crean un ambiente favorable para el desarrollo de F. hepatica, así como para la reproducción de caracoles, principalmente Lymneidos, los cuales abundan en la zona. Estudios paralelos realizados en cerdos, equinos y asnos de Masma reportaron prevalencias de 18.3, 29.4 y $87.7 \%$, respectivamente (Cabrera et al., 2010), permitiendo afirmar que Masma es una zona altamente endémica. Estos aspectos favorecerían considerablemente el mayor riesgo de infección en los niños de este distrito.

E1 11.7\% de prevalencia de fasciolasis en el distrito de Masma fue menor que el 28.3\% reportado por Marcos et al. (2004) en Huertas, pese a que ambas localidades se consideran dentro de una zona hiperendémica. No obstante, esta diferencia podría ser explicada por una menor frecuencia de infección con formas larvarias de F. hepatica en caracoles lymneidos (índices cercarios), donde se halló $0.8 \%(1 / 131)$ y $12.5 \%(11 / 88)$ para Masma y Huertas, respectivamente (A. Chávez, datos no publicados), es decir, un menor nivel de contaminación ambiental con formas infectivas del parásito.

Otro aspecto que explicaría las diferencias de prevalencias en los distritos estudiados, estaría relacionado con indicadores sociodemográficos, como características de las viviendas y características demográficas (poblaciones urbana y rural); así, en el distrito de Masma se observó un mayor número de viviendas construidas con materiales de adobe, donde además era evidente el contacto cercano de la población con las fuentes de agua y sus animales; en tanto que las construcciones en Llocllapampa y Santa Rosa de Ocopa eran de ladrillo y cemento, provistas de agua y de sistema de eliminación de excretas. Estas diferencias colocarían al distrito de Masma como una zona de mayor riesgo, al presentar indicadores de pobreza que reflejan mala nutrición, así como malos hábitos higiénicos y servicios sanitarios deficientes, los cuales constituyen factores antropológicos, sociales y humanos esenciales para la presentación de endemias parasitarias como la fasciolasis (Marcos et al., 2004).

Las bajas prevalencias de fasciolasis humana en los distritos de Santa Rosa (0.9\%) y Llocllapampa ( $0 \%$ ) son similares a los reportados para el distrito del Tambo, en Huancayo (1.3\%) (Ramos, 1991). Estos resultados reflejan el beneficio que aportan las mejoras sanitarias propias de zonas urbanas, 
como son el consumo de agua potable, vegetales lavados y el uso de servicios higiénicos en un 70\% de la población (Ramos, 1991). Por otro lado, en el distrito de Llocllapampa se observó que pocas familias eran propietarias de la ganadería presente en la zona, y los animales eran criados en establos alejados de las viviendas o pastoreados en zonas altas, dado que la principal actividad económica del distrito es la explotación de sílice y mármol.

Se debe resaltar que el método de sedimentación rápida, utilizado en el presente estudio, ha sido comparado con otras técnicas de concentración, tales como la de sedimentación espontánea y de concentración éterformol, mostrando resultados superiores debido al uso de una mayor cantidad de muestra (4-8 g) (Maco et al., 2002). Sin embargo, las técnicas serológicas, como el Fas2-ELISA presenta una sensibilidad del $96.8 \%$ y especificidad del $91.2 \%$, muy superiores al método empleado, de allí que los resultados podrían haber dejado un margen de posibles casos positivos no detectados, debido a la limitación para el diagnóstico de individuos en la fase aguda, la posible localización ectópica del tremátodo, y la eliminación intermitente de huevos por el parásito adulto; así como al uso de una sola muestra fecal en lugar del muestreo seriado.

La semejanza de la prevalencia entre niños y niñas se explicaría por la similitud de las actividades que realizan en el campo (ejemplo: llevar a pastar al ganado) y el acceso común a las fuentes de contaminación, como es el consumo de vegetales crudos o la ingestión de agua contaminada con metacercarias (Marcos et al., 2006). Asimismo, no se halló diferencia significativa entre la prevalencia de la enfermedad y la edad, datos que concuerdan con estudios previos (Apt et al., 1993; Ramos, 1991; Esteban et al., 1997, 1999; Maldonado, 2003; Curtale et al., 2003; Marcos et al., 2004, 2007; Parkinson et al., 2007).
Es importante mencionar que estudios adicionales realizados en bovinos mostraron una elevada frecuencia de fasciolasis, reportando tasas de $0 \%$ (49/100), 41.4\% (48/116) y $47.5 \%$ (56/118) para los distritos de Masma, Llocllapampa y Santa Rosa de Ocopa, respectivamente (A. Chávez, datos no publicados).

\section{Conclusiones}

- La prevalencia de Fasciola hepatica en niños de los distrito de Masma y Santa Rosa de Ocopa fue de $11.7 \pm 5.6$ y $0.9 \pm$ 1.7 , respectivamente. No se hallaron muestras positivas a $F$. hepatica en niños del distrito de Llocllapampa.

- Las variables edad y género no tuvieron una asociación estadística con la presencia de infección en niños de estos distritos.

\section{Literatura Citada}

1. Apt W, Aguilera $X$, Vega $F$, Alcaíno H, Zulantay I, Apt P, Gonzales V, et al. 1993. Prevalencia de fasciolasis en humanos, caballos, cerdos y conejos silvestres en tres provincias de Chile. Bol Ofic Sanit Panam 115: 405-414.

2. Cabrera J, Chávez A, Arana C, Suárez F. 2010. Endoparásitos de suinos y équidos de dos distritos de la provincia de Jauja. En: XXII Congreso Panamericano de Ciencias Veterinarias. Lima.

3. Castro M. 2007. Realidad actual de la distomatosis hepática en animales domésticos del Perú: Revisión de su epidemiología y su frecuencia. Tesina de Médico Veterinario. Lima: Univ Nac Mayor de San Marcos. 86 p.

4. Curtale F, Hassanein YA, Wakeel AE, Mas-Coma S, Montresore A. 2003. Distribution of human fascioliasis by age and gender among rural population in the Nile Delta, Egypt. J Trop Pediatr 49: 264-268. 
5. Daniel W. 1996. Bioestadística base para el análisis de las ciencias de la salud. $3^{a}$ ed. México: Limusa. 875 p.

6. De NV, Hoa LT, Waikagul J. 2006. Plant-borne trematode (fascioliasis) in Vietnam. In: V Seminar on Food- and Water-borne Parasitic Zoonoses. Thailand.

7. Espinoza JR, Terashima A, HerreraVelit P, Marcos LA. 2010. Fasciolasis humana y animal en el Perú: Impacto en la economía de las zonas endémicas. Rev Peru Med Exp Salud Pública 27: 604-612.

8. Esteban JG, Flores A, Aguirre C, Strauss W, Angles R, Mas-Coma S. 1997. Presence of very high prevalence and intensity of infection with Fasciola hepatica among Aymara children from the Northern Bolivian Altiplano. Acta Trop 66: 1-14.

9. Esteban JG, Flores A, Angeles R, Mas-Coma S. 1999. High endemicity of human fasciolasis between Lake Titicaca and La Paz valley, Bolivia. T Roy Soc Trop Med H 93: 151-156.

10. Esteban JG, González C, Bargues MD, Angles R, Sánchez C, Náquira C, Mas-Coma S. 2002. High fascioliasis infection in children linked to a man-made irrigation zone in Perú. Trop Med Int Health 7: 339-348.

11. [INEI] Instituto Nacional de Estadística e Informática. 2007. Censo Nacional: XI de población y VI de vivienda. [Internet]. Disponible en: http:// www.censos.inei.gob.pe/

12. Keiser J, Utzinger J. 2005. Emerging foodborne trematodiasis. Emerg Infect Dis 11: 1507-1514.

13. Leguía G. 1991. Distomatosis hepática en el Perú. Epidemiología y control. 2a ed. Lima: Universidad Nacional Mayor de San Marcos. 45 p.

14. Leguía G. 1997. Parasitary infections of the liver. Rev Gastroenterol Perú 17: 115-127.

15. Lumbreras H, Cantella R, Burga R. 1962. Acerca de un procedimiento de sedimentación rápida para investigar hue- vos de Fasciola hepatica en las heces, su evaluación y uso en el campo. Rev Med Peruana 31: 167-174.

16. Maco F, Marcos R, Terashima A, Samalvides F, Miranda E, Espinoza J, Gotuzzo E. 2002. Fas2 ELISA y la técnica de sedimentación rápida modificada por Lumbreras en el diagnóstico de la infección por Fasciola hepatica. Rev Med Hered 13: 49-57.

17. Maldonado F. 2003. Prevalencia de fasciolasis en una población del distrito de Rahuampa-Ancash. Tesis de Médico Cirujano. Lima: Univ Peruana Cayetano Heredia. 34 p.

18. Marcos LA, Maco V, Terashima A, Samalvides F, Espinoza JR, Gotuzzo E. 2004. Hiperendemicidad de fasciolosis humana en el valle del Mantaro: Factores de riesgo de la infección por Fasciola hepatica. Rev Gastroenterol Perú 24: 158-164.

19. Marcos LA, Maco V, Castillo M, Terashima A, Zerpa R, Gotuzzo E. 2005. Reporte de casos de fasciolosis en el Instituto Especializado de Salud del Niño Lima-Perú (1988-2003). Rev Gastroenterol Perú 25: 198-205.

20. Marcos LA, Maco V, Samalvides F, Terashima A, Espinoza J, Gotuzzo E. 2006. Risk factors for Fasciola hepatica infection in children: a casecontrol study. T Roy Soc Trop Med H 100: 158-166.

21. Marcos LA, Terashima A, Leguía G, Canales M, Espinoza JR, Gotuzzo E. 2007. La Infección por Fasciola hepatica en el Perú: una enfermedad emergente. Rev Gastroenterol Perú 27: 389-396.

22. Mas-Coma S. 2004. Human fascioliasis. In: Cotruvo JA, Dufour A, Rees G, et al. (eds). Waterborne zoonoses: Identification, causes and control. London: IWA Publishing. p 305-322.

23. Mas-Coma S, Esteban JG, Bargues MD. 1999. Epidemiology of human fascioliasis: a review and proposed new classification. Bull WHO 77: 340-346. 
24. [OMS] Organización Mundial de la Salud. 1995. Lucha contra las trematodiasis de transmisión alimentaria. Ginebra: OMS. Serie de Informes Técnicos. $849 \mathrm{p}$.

25. Parkinson M, O'Neill SM, Dalton JP. 2007. Endemic human fasciolosis in the Bolivian Altiplano. Epidemiol Infect 135: 669-674.

26. Pérez C. 2000. Técnicas de muestreo estadístico. México: Alfa Omega. 603 p.

27. Ramos DD. 1991. Fasciolasis hepática en escolares, aspectos epidemiológicos, distrito del Tambo-Huancayo. Tesis de Bachiller en Medicina. Lima: Univ Peruana Cayetano Heredia. 49 p.

28. Stork MG, Venables GS, Jennings SM, Beesley JR, Bendezu P, Capron A.
1973. An investigation of endemic fasciolasis in Peruvian village children. $\mathrm{J}$ Trop Med Hyg 76: 231-235.

29. Terashima MA. 1970. Fasciolasis hepática en escolares de Huertas, Jauja. Consideraciones epidemiológicas, parasitológicas y clínicas. Tesis de Bachiller en Medicina. Lima: Univ Peruana Cayetano Heredia. $37 \mathrm{p}$.

30. Valero MA, Perez-Crespo I, Periago MV, Khoubbane M, Mas-Coma S. 2009. Fluke egg characteristics for the diagnosis of human and animal fascioliasis by Fasciola hepatica and F. gigantica. Acta Trop 111: 150-159.

31. [WHO] World Health Organization. 1991. Basic laboratory methods in medical parasitology. Geneva: WHO. 114 p. 